\title{
Interaction of iron phthalocyanine with the graphene/Ni(111) system
}

\author{
Lorenzo Massimi ${ }^{* 1}$, Simone Lisi ${ }^{1}$, Daniela Pacilè ${ }^{2}$, Carlo Mariani ${ }^{1}$ \\ and Maria Grazia Betti ${ }^{1}$
}

\section{Full Research Paper}

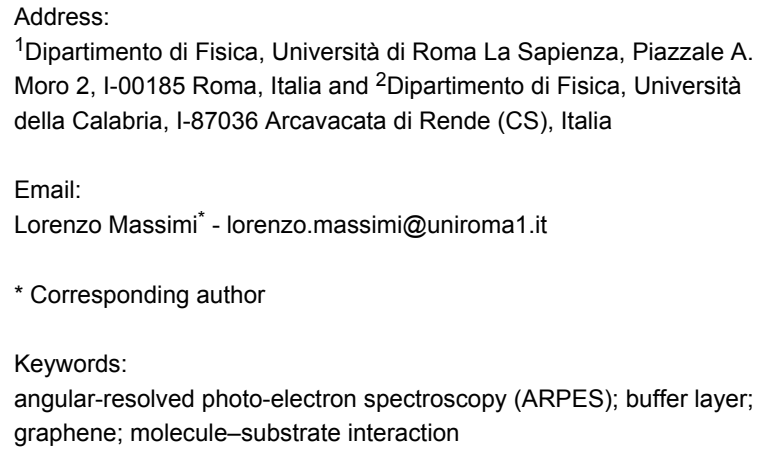

${ }^{1}$ Dipartimento di Fisica, Università di Roma La Sapienza, Piazzale A. Moro 2, I-00185 Roma, Italia and ${ }^{2}$ Dipartimento di Fisica, Università della Calabria, I-87036 Arcavacata di Rende (CS), Italia

Email:

Lorenzo Massimi* - lorenzo.massimi@uniroma1.it

* Corresponding author

Keywords:

angular-resolved photo-electron spectroscopy (ARPES); buffer layer; graphene; molecule-substrate interaction

Open Access

\author{
Beilstein J. Nanotechnol. 2014, 5, 308-312. \\ doi:10.3762/bjnano.5.34 \\ Received: 14 December 2013 \\ Accepted: 17 February 2014 \\ Published: 17 March 2014 \\ This article is part of the Thematic Series "Nanostructures for sensors, \\ electronics, energy and environment II". \\ Guest Editor: N. Motta \\ (C) 2014 Massimi et al; licensee Beilstein-Institut. \\ License and terms: see end of document.
}

\begin{abstract}
Graphene grown on crystalline metal surfaces is a good candidate to act as a buffer layer between the metal and organic molecules that are deposited on top, because it offers the possibility to control the interaction between the substrate and the molecules. Highresolution angular-resolved ultraviolet photo electron spectroscopy (ARPES) is used to determine the interaction states of iron phthalocyanine molecules that are adsorbed onto graphene on $\mathrm{Ni}(111)$. The iron phthalocyanine deposition induces a quenching of the Ni d surface minority band and the appearance of an interface state on graphene/Ni(111). The results have been compared to the deposition of iron phthalocyanine on graphene/Ir(111), for which a higher decoupling of the organic molecule from the underlying metal is exerted by the graphene buffer layer.
\end{abstract}

\section{Introduction}

The interest in the preparation of ordered layers of organic molecules is rapidly growing, because of the possibility to realize advanced electronic- and spin-based devices [1-3]. Transition-metal phthalocyanines (MPcs) are planar molecules that consist of an organic cage formed by four pyrrole and benzene rings with a central metal ion [4]. They represent a class of molecules with potentially large applications thanks to their easily tunable electronic and magnetic properties, which are basically associated with the electronic configuration of the central metal atom [5]. When deposited on surfaces, their inter- action may be driven by dipolar forces mainly related to the organic cage and by a stronger interaction that is associated with the central metal atom. As an example, the adhesion of iron phthalocyanine $(\mathrm{FePc})$ and cobalt phthalocyanine $(\mathrm{CoPc})$ on a Au substrate is mainly due to the presence of unfilled, outof-plane oriented, $d$ states that interact with the underlying gold states $[6,7]$.

The magnetic and electronic properties of the adsorbed molecules may be strongly influenced by the interface and can be 
potentially tuned by using an appropriate buffer layer. Graphene (Gr), thanks to its unique electronic properties [8] and to the quite easy experimental preparation on many metal substrates [9], is a good candidate to tune the MPc-metal interface. Moreover, the self-assembling capabilities of organometallic molecules offer the possibility to form ordered networks of metal atoms trapped in an organic cage, which is a suitable configuration for the realization of spin-based qubits [10]. Interesting and exemplary cases are represented by MPcs adsorbed on graphene grown on $\mathrm{Ni}(111)$ and $\operatorname{Ir}(111)$ surfaces. In fact, graphene on $\mathrm{Ni}(111)$ and on $\operatorname{Ir}(111)$ represents two opposite sides of the graphene-metal interaction: a strong interaction with a strong modification of the free-standing graphene band structure is observed on $\mathrm{Ni}$ [11], while a low interaction with an almost unperturbed Dirac cone is present if graphene is grown on Ir $[12,13]$. Recently it has been shown that graphene acts as a buffer layer that decouples the FePc molecules from $\operatorname{Ir}(111)$ and prevents an Ir-FePc interaction [13]. On the other hand, for $\mathrm{Gr} / \mathrm{Ni}(111)$ a FePc-Ni interaction has been suggested [14-16] despite the presence of the graphene sheet, as it was already observed for $\mathrm{CoPc}$ on $\mathrm{Gr} / \mathrm{Ni}(111)$ [17]. We present a valence band UV photoemission study of the FePc adsorption on $\mathrm{Gr} / \mathrm{Ni}$, which brings to light a direct evidence of an interaction between the FePc molecule and the Ni substrate.

\section{Experimental}

Experiments were performed in situ in ultra-high-vacuum (UHV) chambers with base pressures in the low $10^{-10} \mathrm{mbar}$ range at the LOTUS laboratory of the Università La Sapienza (Roma). The Ni(111) single crystal was cleaned by several sputtering-annealing cycles $\left(1 \mathrm{keV} \mathrm{Ar}{ }^{+}\right.$for $30 \mathrm{~min}, 600{ }^{\circ} \mathrm{C}$ for $10 \mathrm{~min}$ ). Graphene was obtained by exposing the sample, which was kept slightly below $600{ }^{\circ} \mathrm{C}$ to $6000 \mathrm{~L}$ of ethylene ( $1 \mathrm{~L}=10^{-6}$ torr $\left.\cdot \mathrm{s}\right)$. The formation of graphene on the $\mathrm{Ni}(111)$ surface is complicated by the segregation of carbon from the bulk, because of the high solubility of carbon in $\mathrm{Ni}$ [18-20]. The $\operatorname{Ir}(111)$ single crystal was cleaned by several sputtering-annealing cycles $\left(2 \mathrm{keV} \mathrm{Ar}{ }^{+}\right.$for $30 \mathrm{~min}, 1200{ }^{\circ} \mathrm{C}$ for $60 \mathrm{~s})$. The preparation of graphene was done by several 120 -seconds long exposures to ethylene while flash-heating the sample up to $1100^{\circ} \mathrm{C}$.

The deposition of FePc was carried out by using a custom-made quartz crucible and the molecular deposition was controlled by using a quartz microbalance. One single-layer (SL) is defined as the molecular density of flat molecules fully covering the graphene layer, and it corresponds to a nominal thickness of about $3.4 \AA$.

Low energy electron diffraction (LEED) was used to check the symmetry of both clean and Gr-covered surfaces. LEED was performed in the energy range of the primary beam of 90-140 eV. High-resolution angular-resolved photoelectron spectroscopy data was carried out by using a SCIENTA SES200 analyzer with an angular acceptance of $\pm 8^{\circ}$ and a resolution of $16 \mathrm{meV}$. All spectra have been taken along the $\Gamma \mathrm{K}$ direction. The UV radiation, $\mathrm{HeI} \alpha(21.218 \mathrm{eV})$ and $\mathrm{HeII} \alpha$ $(40.814 \mathrm{eV})$, was provided by a SCIENTA VUV-5050 monochromatic source.

\section{Results and Discussion}

The ARPES band structure of the Gr/Ni(111) and Gr/Ir(111) systems along the $\Gamma \mathrm{K}$ direction of the two-dimensional (2D) Brillouin Zone (BZ) is presented in Figure 1. The corresponding LEED patterns are shown in the insets: for $\mathrm{Gr} / \mathrm{Ni}(111)$ the graphene lattice is well aligned with the substrate and no corrugation is present, with a resulting $(1 \times 1)$ symmetry $[21,22]$, while in $\mathrm{Gr} / \operatorname{Ir}(111)$ the lattice mismatch, reflected in the additional moiré pattern, introduces a large-scale regular corrugation [23].

The presence of a distinct single $\pi$ band for both Gr sheets (on $\mathrm{Ni}$ and on Ir) reveals the single-layer nature of graphene. For $\mathrm{Gr} / \mathrm{Ir}(111)$, the Dirac point is localized on a projected bulk band gap and the graphene $\pi$ band looks very similar to the band of free-standing graphene, the linear dispersion of the $\pi$ band is preserved close to the $\mathrm{K}$ point with only a slight $\mathrm{p}$-doping, which is in agreement with the literature $[12,13]$. The very tiny doping has also been interpreted as slight hybridization between the Gr- $\pi$ states and the underlying Ir $\mathrm{d}$ bands, which leads to a gap with a width of a few tens of meV [24]. The small size of the gap can be explained by the small difference among the two sublattices, due to the low interaction with the substrate. The band structure of the $\mathrm{Gr} / \mathrm{Ni}(111)$ system appears to be dominated by the strong projected Ni d bands very close to the Fermi level. Furthermore, the Gr- $\pi$ band is shifted by $2.5 \mathrm{eV}$ towards higher binding energies as compared to $\mathrm{Gr} / \mathrm{Ir}$, and no linear dispersion is observed at the $\mathrm{K}$ point, which is in agreement with previous results [11]. Carbon atoms adsorb on two different sites on $\mathrm{Ni}(111)$, on top of Ni surface atoms and in fcc-hollow sites of the underlying Ni mesh [21,25]. As a consequence of the different adsorption sites and of the strong interaction, a large asymmetry among the two carbon sublattices is introduced, which induces a band-gap opening [11]. Recent experiments performed along the BZ direction perpendicular to $\Gamma K$ confirm the strong shift of the Dirac point, while the gap opening is attributed to a strong hybridization of the Gr- $\pi^{*}$ states with the Ni d bands [22].

The photoemission data in the low binding energy region for the iron phthalocyanine molecules deposited on the Gr/Ni surface, and on $\mathrm{Gr} / \mathrm{Ir}$ for comparison, as a function of the thickness of 


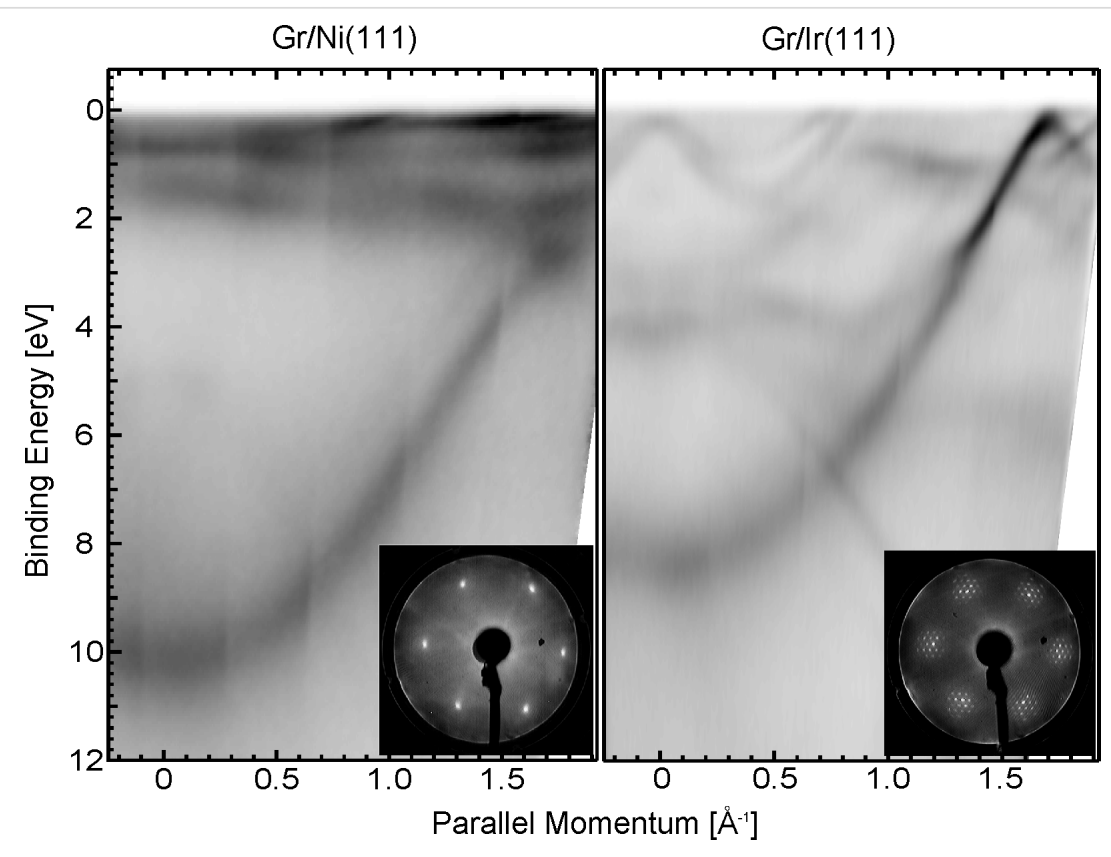

Figure 1: Experimental ARPES band structure for graphene grown on Ni(111) (left) and on $\operatorname{Ir}(111)$ (right), taken with $40.814 \mathrm{eV}$ photon energy along the $\Gamma K$ direction of the 2D BZ. Insets: corresponding LEED patterns taken on $\mathrm{Gr} / \mathrm{Ni}$ and $\mathrm{Gr} / \mathrm{Ir}$, at primary beam energies of $90 \mathrm{eV}$ and $140 \mathrm{eV}$, respectively. LEED patterns have been obtained by using a different geometry.

the FePc layer is shown in Figure 2. The FePc adsorption on $\mathrm{Gr} / \mathrm{Ni}(111)$ produces a general reduction of the prevalent $\mathrm{d}$ band spectral density of states and a new feature emerges close to the
Fermi level (at about $0.3 \mathrm{eV} \mathrm{BE}$ ). Its intensity grows upon increasing the molecular coverage up to completion of the first SL, and starts to decrease at higher thicknesses, a behaviour

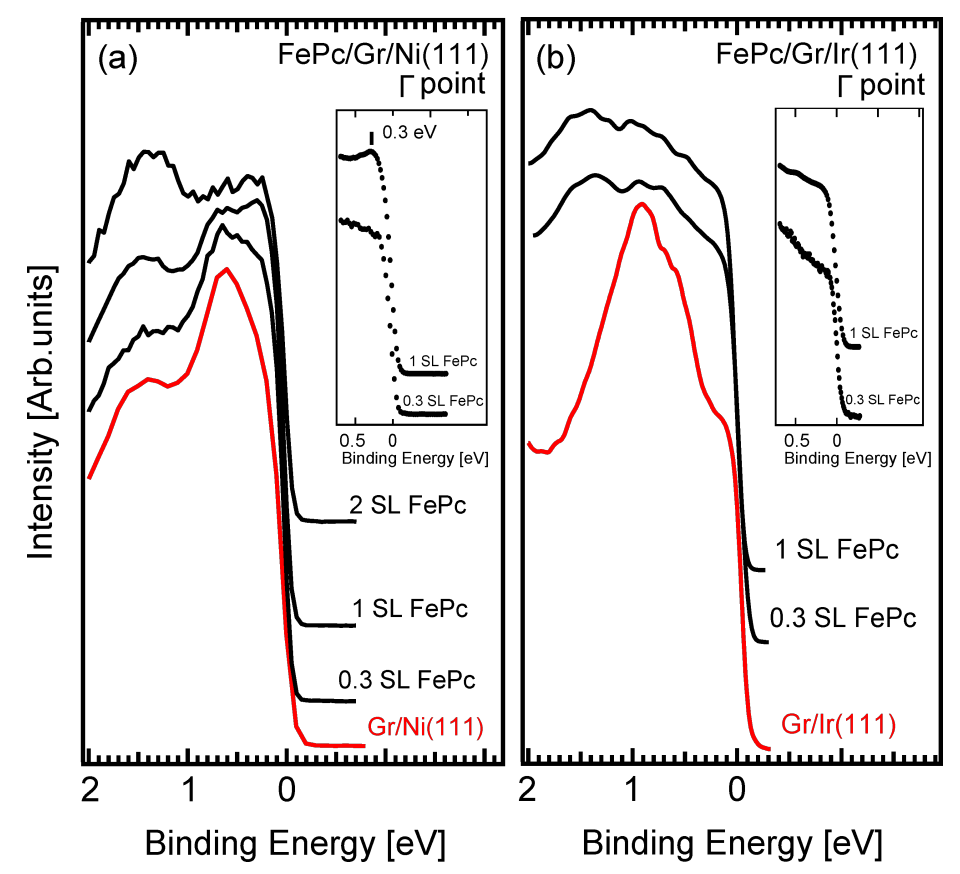

Figure 2: Valence band photoemission data for the adsorption of $\mathrm{FePc}$ onto $\mathrm{Gr} / \mathrm{Ni}(\mathrm{a})$ and onto $\mathrm{Gr} / \mathrm{lr}(\mathrm{b})$, as a function of the thickness of $\mathrm{FePc}$ layer. Data of clean graphene (red lines) and of the FePc/Gr systems (black lines). Data taken with $40.814 \mathrm{eV}$ photon energy (Hell $\alpha$ ) and around normal emission ( $\pm 4^{\circ}$ angular integration around the $\Gamma$ point). The data was normalized to the intensity at the Fermi edge and vertically stacked for clarity. In the insets, a zoom around the Fermi level for a coverage of 0.3 and $1 \mathrm{SL}$ of FePc is given. 
typical of an interface state. This interface state comes from charge transfer from the $\mathrm{Gr} / \mathrm{Ni}$ substrate to partially filled $\mathrm{d}$ orbitals of the central metal ion [15]. On the other hand, the deposition of $\mathrm{FePc}$ on the $\mathrm{Gr} / \mathrm{Ir}$ surface causes only a general attenuation of the Gr/Ir spectral density, while no new state emerges, which confirms previous results [13]. The presence of an interface state for $\mathrm{FePc}$ adsorbed on $\mathrm{Gr} / \mathrm{Ni}$ but not for $\mathrm{FePc}$ adsorbed on $\mathrm{Gr} / \mathrm{Ir}$ can be very likely related to an interaction of the FePc molecules with the substrate underlying the graphene layer. In fact, the shorter distance of $\mathrm{Gr}-\mathrm{Ni}$ compared to $\mathrm{Gr}-\mathrm{Ir}$ $[25,26]$ may induce a larger overlapping of the partially empty out-of-plane d like orbitals of FePc with the hybridized $d-\pi$ states of $\mathrm{Ni}-\mathrm{Gr}$.

In order to better understand the nature of the interaction, we also analyze data at the $\mathrm{K}$ point of the BZ, as shown in Figure 3. At the clean Ni(111) surface (Figure 3, bottom spectrum), we observe two main peaks close to the Fermi level, at $0.08 \mathrm{eV}$ and $0.30 \mathrm{eV} \mathrm{BE}$, respectively. As it is well known, the first one is attributed to d electron minority spin with sp-contribution and the second one to the majority spin [27]. The formation of graphene onto $\mathrm{Ni}(111)$ induces an increase in intensity accompanied by a very slight shift $(-0.02 \mathrm{eV})$ of the first feature, while the $\mathrm{d}$ majority band at $0.3 \mathrm{eV} \mathrm{BE}$ remains unchanged and shows only a slight intensity reduction. The observed change of the minority $\mathrm{d}$ band originates from a hybridization with the

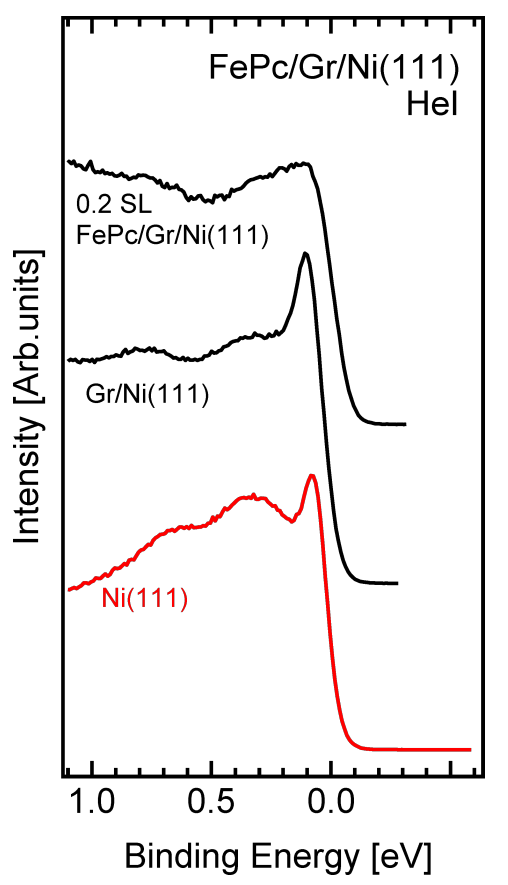

Figure 3: Valence band spectral density of states of clean $\mathrm{Ni}(111)$ (red line), of $\mathrm{Gr} / \mathrm{Ni}(111)$ and of $0.2 \mathrm{SL} \mathrm{FePc}$ onto $\mathrm{Gr} / \mathrm{Ni}$ (black lines), taken at the $\mathrm{K}$ point of the $\mathrm{BZ}\left( \pm 2^{\circ}\right.$ angular integration around $\mathrm{K}$, with $21.218 \mathrm{eV}$ photon energy). graphene $\pi$ bands [25]. The increased intensity of the lowest BE peak is emphasized by the high excitation cross-section for the $\mathrm{C} \pi$-bands with respect to the Ni d-like states [28], which brings to light the hybrid nature of this first peak. Upon adsorption of a tiny quantity of FePc, this hybrid state is strongly reduced in intensity, while the d majority band appears to be basically unaffected. The strong reduction in intensity of the $\pi-\mathrm{d}$ hybrid state suggests a molecule interaction with the $\mathrm{Gr} / \mathrm{Ni}(111)$ interface, which validates the suggestion of a molecule-substrate interaction that is mediated by graphene. This is in agreement with recent investigations, in which electron energy loss and photoemission spectroscopy were used $[14,15]$.

\section{Conclusion}

When used as a buffer layer between an organic molecule and a metal surface, graphene plays a different role in the molecule-metal interaction that depends on the interaction of graphene with the metal substrate. Graphene on Ni(111) reveals a strong interaction with the substrate and strong alteration of the ideal graphene $\pi$ band. After deposition of small amounts of FePc molecules, by means of high-resolution UV photoemission we give direct experimental evidence of an interaction of the molecule with Ni through graphene, as shown by the emerging of an interface state at about $0.3 \mathrm{eV}$ binding energy in normal emission and by the quenching of the Gr-Ni $\pi-\mathrm{d}$ hybrid state at the K point of the BZ.

\section{Acknowledgements}

Work funded by PRIN grant 20105ZZTSE "GRAF" and by FIRB-Futuro in Ricerca 2010-Project PLASMO-GRAPH of the Italian Ministery for Research (MIUR) and by Roma "La Sapienza" University funds.

\section{References}

1. Bogani, L.; Wernsdorfer, W. Nat. Mater. 2008, 7, 179-186. doi:10.1038/nmat2133

2. Coe, S.; Woo, W.-K.; Bawendi, M.; Bulović, V. Nature 2002, 420, 800-803. doi:10.1038/nature01217

3. Bao, Z.; Lovinger, A. J.; Dodabalapur, A. Appl. Phys. Lett. 1996, 69, 3066-3068. doi:10.1063/1.116841

4. Forrest, S. R. Chem. Rev. 1997, 97, 1793-1896. doi:10.1021/cr941014o

5. Liao, M.-S.; Scheiner, S. J. Chem. Phys. 2001, 114, 9780. doi:10.1063/1.1367374

6. Gargiani, P.; Angelucci, M.; Mariani, C.; Betti, M. G. Phys. Rev. B 2010, 81, 085412. doi:10.1103/PhysRevB.81.085412

7. Betti, M. G.; Gargiani, P.; Frisenda, R.; Biagi, R.; Cossaro, A.; Verdini, A.; Floreano, L.; Mariani, C. J. Phys. Chem. C 2010, 114, 21638-21644. doi:10.1021/jp108734u

8. Geim, A. K.; Novoselov, K. S. Nat. Mater. 2007, 6, 183-191. doi:10.1038/nmat1849

9. Batzill, M. Surf. Sci. Rep. 2012, 67, 83-115. doi:10.1016/j.surfrep.2011.12.001 
10. Avdoshenko, S. M.; loffe, I. N.; Cuniberti, G.; Dunsch, L.; Popov, A. A. ACS Nano 2011, 5, 9939-9949. doi:10.1021/nn203719a

11. Dedkov, Y. S.; Fonin, M. New J. Phys. 2010, 12, 125004. doi:10.1088/1367-2630/12/12/125004

12. Pletikosic, I.; Kralj, M.; Pervan, P.; Brako, R.; Coraux, J.; N'diaye, A.; Busse, C.; Michely, T. arXiv preprint arXiv:0807.27702008.

13. Scardamaglia, M.; Lisi, S.; Lizzit, S.; Baraldi, A.; Larciprete, R.; Mariani, C.; Betti, M. G. J. Phys. Chem. C 2013, 117, 3019-3027. doi:10.1021/jp308861b

14. Dou, W.; Huang, S.; Zhang, R.; Lee, C. J. Chem. Phys. 2011, 134, 094705. doi:10.1063/1.3561398

15. Wei-Guo, Y.; Dan, L.; Xiao-Feng, P.; Wei-Dong, D. Chin. Phys. B 2013, 22, 117301. doi:10.1088/1674-1056/22/11/117301

16. Dou, W.; Yang, Q.; Lee, C.-S. Appl. Phys. Lett. 2013, 102, 131606. doi:10.1063/1.4800235

17. Uihlein, J.; Peisert, H.; Glaser, M.; Polek, M.; Adler, H.; Petraki, F.; Ovsyannikov, R.; Bauer, M.; Chassé, T. J. Chem. Phys. 2013, 138, 081101. doi:10.1063/1.4793523

18. Grüneis, A.; Kummer, K.; Vyalikh, D. V. New J. Phys. 2009, 11, 073050. doi:10.1088/1367-2630/11/7/073050

19. Lahiri, J.; Miller, T. S.; Ross, A. J.; Adamska, L.; Oleynik, I. I.; Batzill, M. New J. Phys. 2011, 13, 025001. doi:10.1088/1367-2630/13/2/025001

20. Patera, L. L.; Africh, C.; Weatherup, R. S.; Blume, R.; Bhardwaj, S. Castellarin-Cudia, C.; Knop-Gericke, A.; Schloegl, R.; Comelli, G.; Hofmann, S.; Cepek, C. ACS Nano 2013, 7, 7901-7912. doi:10.1021/nn402927q

21. Gamo, Y.; Nagashima, A.; Wakabayashi, M.; Terai, M.; Oshima, C Surf. Sci. 1997, 374, 61-64. doi:10.1016/S0039-6028(96)00785-6

22. Varykhalov, A.; Marchenko, D.; Sánchez-Barriga, J.; Scholz, M.; Verberck, B.; Trauzettel, B.; Wehling, T.; Carbone, C.; Rader, O. Phys. Rev. X 2012, 2, 041017. doi:10.1103/PhysRevX.2.041017

23. N'Diaye, A. T.; Coraux, J.; Plasa, T. N.; Busse, C.; Michely, T. New J. Phys. 2008, 10, 043033. doi:10.1088/1367-2630/10/4/043033

24. Starodub, E.; Bostwick, A.; Moreschini, L.; Nie, S.; El Gabaly, F.; McCarty, K. F.; Rotenberg, E. Phys. Rev. B 2011, 83, 125428. doi:10.1103/PhysRevB.83.125428

25. Bertoni, G.; Calmels, L.; Altibelli, A.; Serin, V. Phys. Rev. B 2005, 71, 075402. doi:10.1103/PhysRevB.71.075402

26. Sun, Z.; Hämäläinen, S. K.; Sainio, J.; Lahtinen, J.; Vanmaekelbergh, D.; Liljeroth, P. Phys. Rev. B 2011, 83, 081415. doi:10.1103/PhysRevB.83.081415

27. Kreutz, T. J.; Greber, T.; Aebi, P.; Osterwalder, J. Phys. Rev. B 1998, 58, 1300. doi:10.1103/PhysRevB.58.1300

28. Yeh, J.; Lindau, I. At. Data Nucl. Data Tables 1985, 32, 1-155. doi:10.1016/0092-640X(85)90016-6

\section{License and Terms}

This is an Open Access article under the terms of the Creative Commons Attribution License

(http://creativecommons.org/licenses/by/2.0), which permits unrestricted use, distribution, and reproduction in any medium, provided the original work is properly cited.

The license is subject to the Beilstein Journal of Nanotechnology terms and conditions: (http://www.beilstein-journals.org/bjnano)

The definitive version of this article is the electronic one which can be found at:

doi:10.3762/bjnano.5.34 